\title{
A case of posterior reversible encephalopathy syndrome
}

\author{
Authors: R Chhabra and C Magee
}

\section{Aims}

Discussion of a rare case of posterior reversible encephalopathy syndrome (PRES), a clinical radiological syndrome characterised by headache, confusion, visual disturbance and seizures associated with typical neuroimaging findings of posterior cerebral white matter oedema.

\section{Methods}

We report a case of a 74-year-old lady who was electively admitted for initiation of haemodialysis for symptomatic volume overload.

Her background history was significant for end-stage kidney disease and nephrotic syndrome secondary to membranous nephropathy, for which she was recently treated with cyclophosphamide and steroids. She had been recently treated for herpes zoster ophthalmicus.

She had three sessions of uneventful dialysis over three consecutive days. However, subsequent development of malignant hypertension was noted post dialysis. She subsequently developed tonic-clonic seizures associated with a drop in GCS to 9 and a low-grade temperature.

Broad-spectrum antimicrobial cover was initiated given her immunosuppressed state. An urgent CT brain scan revealed findings suspicious of PRES. MRI of her brain showed bilateral cortical and subcortical white matter symmetric signal abnormality in the occipital, parietal and superior frontal lobes, confirming a diagnosis of PRES.

Labetalol infusion was commenced to achieve a slow reduction in blood pressure (BP) to a target of $<140 / 90 \mathrm{mmHg}$.

\section{Results}

She made a remarkable recovery with good BP control. A repeat CT brain scan in 2 weeks showed a significant improvement.

\section{Conclusions}

PRES is a rare entity and should be considered in a hypertensive patient who develops headache, visual disturbance or confusion. Most cases are fully reversible with BP control and treating the underlying cause. 